\title{
Investigation of the Relationship Between Exposure to Preeclampsia and Overweight/Obesity in 2-7 Year Old Children
}

\author{
Mahshid Ahmadi $^{(\mathbb{D}}$, Jila Ganji ${ }^{2,3^{*}}{ }^{\mathbb{D}}$, Daniel Zamanfar ${ }^{4}$, Parisa Ghobadi Golafshani ${ }^{5}$
}

\begin{abstract}
Objectives: Childhood obesity has reached alarming and concerning levels in many countries and poses an urgent and serious challenge to public health. In addition, gestational hypertension contributes to an elevated risk of obesity in children. The present study aimed at determining the relationship between exposure to preeclampsia and overweight/obesity occurrence in children aged 2-7 years old.

Materials and Methods: This observational case-control study was designed to assess the relationship between exposure to preeclampsia and overweight/obesity in children aged 2-7 years old. The study population included 205 children within the age range of 2-7 years old referring to Sari Pediatric Endocrinology Clinic as the case group and 209 healthy children who were selected from health centers as the control group and were homogenous in terms of demographic variables. Finally, data were analyzed in SPSS software (version 24), and P value of less than 0.05 was considered statistically significant.

Results: The present study was conducted on a total number of 414 children aged 2-7 years old with a mean and standard deviation of $4.41 \pm 1.76$ years, including 226 females (54.6\%) and 188 males (45.4\%). The obtained results revealed that gestational hypertension increased the risk of obesity in children significantly ( $\mathrm{OR}=1.88,95 \% \mathrm{CI}=1.46-2.68)$.

Conclusions: Based on the results of the present research and similar studies, gestational hypertension is associated with overweight and obesity in children.

Keywords: Preeclampsia, Gestational hypertension, Child, Obesity, Overweight
\end{abstract}

\section{Introduction}

Obesity in children is one of the uttermost urgent and critical public health challenges in the 21 st century in many countries (1). Based on reports presented on World Obesity Day (2017), overweight/obesity rates have risen 10 -fold over the recent four decades. Based on a systematic review and meta-analysis study in 2014, the overall prevalence of obesity/overweight in Iranian children and adolescents was $10.8 \%$ and $5.1 \%$, respectively $(2,3)$. The child's biological responses to obesity-prone environments are shaped by some processes even before birth. Therefore, fighting the growing obesity epidemic requires addressing factors from pre-fertilization to late childhood (4).

Numerous studies have so far focused on high-risk pregnancies, especially preeclampsia and its consequences on children $(5,6)$. This disease, which is described by hypertension and proteinuria, influences 3\%-5\% of all pregnancies worldwide (7). Prenatal exposure to preeclampsia may result in prematurity, low birth weight, increased growth, and body mass index (BMI) in infancy and adolescence (8). Researchers attribute various complications of preeclampsia to the release of antiangiogenic factors as a result of placental insufficiency. They have further hypothesized that hunger in the prenatal period is associated with epigenetic alterations which remain during the entire life and lead to the tendency to conserve energy and obesity (9-12).

Various studies have so far evaluated the adverse effects of high-risk pregnancies on children, as well as the relationship between maternal preeclampsia and childhood obesity. However, this association has not been clearly elucidated, has not been meaningful, or has yielded conflicting results in terms of age, gender, and the severity of exposure (13-19). On the other hand, no related research has yet been conducted in Iran. Accordingly, the present study was performed to investigate the relationship between preeclampsia and overweight/obesity in children aged 2-7 years old.

\section{Materials and Methods}

This is an observational-analytical study with a case- 
Key Messages

- The obtained results revealed that gestational hypertension increased the risk of obesity in children significantly.

- The results of the current study has clinical implication regarding care of children born to preeclamptic mothers.

control design. The sample size was calculated according to Zheng's study (18). The current study investigated the relationship between exposure to maternal preeclampsia and overweight/obesity in 2-7-year-old children. The study population included the case and control groups. The case group consisted of 205 children aged 2-7 years old, who referred to Sari Pediatric Endocrinology Clinic. On the other hand, the control group included 209 children who were healthy and homogenous in terms of demographic characteristics and were selected from health centers.

First, the archives of obese children at the endocrinology clinic were searched for the list of obese or overweight children aged 2-7 years old, along with their contact numbers. Then, the researchers contacted children's families and introduced a research plan and procedure. In addition, the written consent was obtained from all those who agreed to participate, and they were assured of the confidentiality of their responses. Subsequently, data collection forms were administered to the participants and the responses were recorded accordingly. The questions included data about the parent' occupation, family incomes, parent's weight and height, history of obesity in other children, history of pregnancy and delivery, childbirth history, type of infant feeding, initiation and type of supplementary nutrition, children disease, use of medications in children, the place for keeping children, the number of child caretakers, and the child's activity level (20).

Next, the information of the health care center was obtained to minimize the information error in the control group and to obtain the needed information from the medical record of the child's family. Thereafter, permission was taken from Sari Health Center to access the information of health centers. The researchers then referred to the relevant center, presented their research plan to the expert of the family health unit, and selected some children for the control group. The children had normal BMI and were homogenous with the case group in terms of age, gender, mother's weight, and social-economic status. Next, the questionnaires were administered to them and the obtained information was recorded. In the case of the unreliability of the mother's reports or non-access to her records (gestational hypertension), the needed information was extracted from the documentation in the medical record of the family (family doctor).

The case group included children within the age range of 2 years ( 1 year, 11 months, and 29 days) to 7 years old (6 years, 11 months, and 29 days) which fell into the obesity category $(>+3 \mathrm{Z}$-score) in the BMI/Age WHO chart. Children within the age range of 2 years (1 year, 11 months, and 29 days) to 7 years (6 years, 11 months, and 29 days) with $+2 \mathrm{Z}$-score $<\mathrm{BMI} \leq+3 \mathrm{Z}$ score in the BMI/ Age WHO chart were allocated to the overweight group. Moreover, the control group included children within the age range of 2 years ( 1 year, 11 months, and 29 days) to 7 years (6 years, 11 months, and 29 days) with a normal BMI (-2Z-score $\leq \mathrm{BMI} \leq+1 \mathrm{Z}$-score) in the $\mathrm{BMI} /$ Age WHO chart (21). It should be noted that children in this group were homogenous with the case group in terms of age and gender.

\section{Inclusion Criteria}

- A 2-7-year-old child with obesity referring to the pediatric endocrinology clinic;

- A 2-7-year-old child with overweight referring to the pediatric endocrinology clinic.

\section{Exclusion Criteria}

- Obesity-producing diseases (confirmed by a pediatrician) such as Cushing's syndrome, hypothyroidism, growth hormone deficiency, and hypothalamic obesity;

- Use of some drugs such as anticonvulsants and corticosteroids;

- Maternal history of chronic diseases, especially primary and secondary hypertension and diabetes mellitus;

- Mother's lack of care for the child or child care by several people.

Exposure included maternal preeclampsia during pregnancy. According to the National Guidelines for Obstetrics and Gynecology Services (22), preeclampsia is defined as blood pressure $\geq 140 / 90 \mathrm{~mm} \mathrm{Hg}$ plus proteinuria which is screened during each pregnancy care by measuring the blood pressure in a fixed position (sitting or lying down) and the right or left hand (23).

Finally, the data were analyzed by SPSS software (version 24) using the mean difference, or frequency distribution of quantitative and qualitative variables between the two groups using the independent $t$ test, Mann-Whitney $\mathrm{U}$ test, and chi-square test. In addition, logistic regression and related modeling were used to estimate point and distance (95\% confidence interval) odds ratio of maternal preeclampsia. They were performed by entering all raw variables and then by adjustments for confounding variables. A $P$-value of less than 0.05 was considered statistically significant.

\section{Results}

A total number of 414 children participated in the present study (Table 1).

The assessment of the history of miscarriage and death of previous neonates, as well as pediatric diseases indicated 
that $65(15.7 \%)$ and $13(3.1 \%)$ mothers had a history of miscarriage and child death, respectively. In addition, the history of obesity in other children of the family was positive in 36 cases $(8.7 \%)$.

Investigation of other variables related to maternal delivery demonstrated that 317 women (76.6\%) had a cesarean section and the remaining cases had a vaginal delivery. Only four mothers (1.0\%) had multiple pregnancies, 391 cases (94.4) had full-term delivery, and 40 of them (9.7\%) had gestational hypertension.

The highest frequency concerning the number of children was reported as 2 children in 183 of cases (44.2\%), followed by 1 child in 180 cases $(43.5 \%)$. Moreover, the highest number of deliveries was 2 in 194 cases (46.9\%) and the highest frequency of pregnancies was reported as two pregnancies in 188 cases (45.4\%). The study of variables related to newborns' nutrition indicated that 343 (82.9\%), 24 (5.8\%), and 47 (11.4\%) cases were exclusively breastfed, formula-fed, and both breastfed and formula-

Table 1. Demographic Characteristics of Overweight/Obese Children Aged 2-7 Years Old

\begin{tabular}{|c|c|c|c|}
\hline \multirow{2}{*}{ Variable } & \multicolumn{3}{|c|}{ Total Participants } \\
\hline & Minimum & Maximum & Mean \pm SD \\
\hline Child's age (y) & 2 & 7 & $76.1 \pm 41.4$ \\
\hline Child's weight (kg) & 10 & 53 & $59.20 \pm 18.8$ \\
\hline Child's height & 80 & 142 & $106.37 \pm 14.32$ \\
\hline BMI & 13 & 46 & $17.64 \pm 3.71$ \\
\hline Father's age & 23 & 73 & $35.35 \pm 5.98$ \\
\hline Mother's age & 18 & 48 & $32.22 \pm 5.57$ \\
\hline \multirow[t]{2}{*}{ Mother's BMI } & 18 & 51 & $29.19 \pm 5.21$ \\
\hline & & & No. (\%) \\
\hline \multirow{2}{*}{ Gender } & \multicolumn{2}{|l|}{ Female } & $222(54.6)$ \\
\hline & \multicolumn{2}{|l|}{ Male } & $188(45.4)$ \\
\hline \multirow{2}{*}{$\begin{array}{l}\text { Father's } \\
\text { occupation }\end{array}$} & \multicolumn{2}{|c|}{ Unemployed } & $69(16.7)$ \\
\hline & \multicolumn{2}{|l|}{ Employed } & $345(83.3)$ \\
\hline \multirow{2}{*}{$\begin{array}{l}\text { Mother's } \\
\text { occupation }\end{array}$} & \multicolumn{2}{|l|}{ Housewife } & $376(90.8)$ \\
\hline & \multicolumn{2}{|l|}{ Employed } & $38(9.2)$ \\
\hline \multirow{3}{*}{$\begin{array}{l}\text { Monthly family } \\
\text { income }\end{array}$} & \multicolumn{2}{|c|}{$<1$ million toman } & $121(29.2)$ \\
\hline & \multicolumn{2}{|c|}{ 1-3 million toman } & $266(64.3)$ \\
\hline & \multicolumn{2}{|c|}{ >3 million toman } & $27(6.5)$ \\
\hline
\end{tabular}

Note. SD: Standard deviation; BMI: Body mass index. fed, respectively. Supplementary feeding was according to the national plan in 319 cases $(77.1 \%)$. Nonetheless, solid foods initiated before 6 months of age in 35 cases (8.5\%).

The results of this study showed that the highest frequency of watching television or playing computer games on a daily basis was measured at 1 hour to 2 hours in 319 cases $(77.1 \%)$, followed by more than 2 hours in 60 cases (14.5\%). Moreover, caretakers were parents in most cases. Additionally, the assessment of quantitative variables between normal and overweight children demonstrated that BMI was obtained at $15.65 \pm 2.70$ and $19.66 \pm 3.51$ in normal and overweight children with a statistically significant difference, respectively $(P>0.0001)$. Maternal weight and BMI were also higher in overweight children, this difference was statistically significant $(P<0.0001)$.

Maternal weights at the beginning of pregnancy were $68.53 \pm 12.87$ and $75.79 \pm 13.79$ in normal and overweight groups, respectively. This difference was also statistically significant $(P<0.0001)$. Further, normal neonates had a mean weight of $3296.9 \pm 503.8$ whereas overweight neonates had a mean weight of $3401.51 \pm 543.39$, signifying a significant difference in this regard $(P=0.043)$. However, no statistically significant difference was considered in other quantitative variables $(P>0.05)$ (Table 2$)$.

Examination of demographic variables among normal and overweight children revealed that the frequency of females in normal and overweight groups was 101 (48.3\%) and $125(161.05 \%)$ cases, respectively. On the other hand, the frequency of males in these two groups was 108 (51.7\%) and $80(39.0 \%)$ cases, respectively. As shown, this discrepancy is significant statistically $(P=0.010)$. In addition, $18(8.6 \%)$ and 51 (24.9\%) unemployed fathers were reported in normal and overweight groups, respectively, which suggested a statistically significant difference $(P>0.05)$.

The history of abortion, obesity, the mortality of other children, and children's disease were assessed as well. In this regard, the history of child death was detected in 2 $(1.0 \%)$ and $11(5.4 \%)$ cases in normal weight and case groups, respectively, indicating a statistically significant difference $(P=0.0010)$. Furthermore, the history of obesity in other children of the control group was reported

Table 2. Evaluation of Quantitative Variables in Overweight/Obese Children Aged 2-7 Years Old in Case and Control Groups

\begin{tabular}{llll}
\hline Variable & Control Group & Case Group & P Value \\
\hline Body mass index & $15.56 \pm 2.70$ & $19.66 \pm 3.51$ & $<0.0001$ \\
Father's age & $35.31 \pm 6.53$ & $35.39 \pm 5.37$ & 0.893 \\
Mother's age & $32.44 \pm 5.59$ & $32.00 \pm 5.60$ & 0.422 \\
Mother's height $(\mathrm{cm})$ & $160.31 \pm 5.30$ & $161.38 \pm 6.14$ & 0.059 \\
Mother's weight $(\mathrm{kg})$ & $72.57 \pm 13.24$ & $78.68 \pm 14.96$ & $<0.0001$ \\
Mother's body mass index & $28.21 \pm 4.83$ & $30.19 \pm 5.40$ & $<0.0001$ \\
Maternal age on pregnancy & $28.00 \pm 5.45$ & $27.00 \pm 5.34$ & 0.061 \\
Maternal weight on pregnancy & $68.53 \pm 12.87$ & $75.79 \pm 13.79$ & $<0.0001$ \\
Neonate birth weigh & $3296.99 \pm 503.83$ & $3401.51 \pm 543.39$ & 0.043 \\
\hline
\end{tabular}


in 2 cases $(1.0 \%)$ whereas it was found in 34 cases $(16.6 \%)$ in the overweight group, this difference was statistically significant $(P<0.0001)$. Additionally, the assessment of pregnancy and delivery variables in both groups indicated that $8(3.8 \%)$ and 32 mothers (15.6\%) had gestational hypertension in control and case groups, respectively, which demonstrates a significant difference in this regard $(P=0.02)$. Nonetheless, there was no significant difference between the two groups concerning other variables $(P>0.05)$.

Among the variables related to nutrition, solid food feeding before 6 months of age was reported in 11 (5.3\%) and $24(11.7 \%)$ cases in normal and overweight groups, respectively, showing a significant difference $(P=0.018)$. Nevertheless, there was no significant difference regarding other variables $(P<0.05)$.

Based on the results, 194 (92.8\%) and 125 (61.0\%) children watched television or played video games for 1 hour to 2 hours in normal and overweight groups, respectively. Moreover, 15 (7.2\%) and $45(22.0 \%)$ cases spent more than 2 hours watching television or playing computer games in normal and overweight groups, respectively, which indicated a significant difference $(P<0.0001)$. Contrarily, no significant difference was observed in the type of childcare between the two groups. Based on the results of the assessment of the variables in logistic regression analysis, gestational hypertension significantly increased the odds ratio of obesity in children $(\mathrm{OR}=1.88,95 \% \mathrm{IC}=1.46-2.68)$. Moreover, the odds ratios of obesity for the history of obesity in other children and the history of child disease were reported as 20.58 (CI $95 \%=1.06-8.46)$, 3.37 (95\% CI =1.54-7.36), respectively. This value was obtained at $2.99(95 \% \mathrm{CI}=1.06-8.46)$ and 2.39 (95\% CI $=1.14-5.01)$ for the history of medication use in children and the initiation of solid complementary food before 6 months of age. Eventually, the odds ratio of obesity for pre-term or post-term delivery was calculated at $3.06(95 \% \mathrm{CI}=1.18-7.92)$ (Table 3$)$.

\section{Discussion}

The findings of the present study showed that gestational hypertension significantly increased the odds ratio of obesity in children $(\mathrm{OR}=1.99,95 \% \mathrm{CI}=1.46-2.68)$. This finding is in line with the results of a prospective cohort study performed by Zheng et al that demonstrated a positive correlation between diastolic and systolic blood pressure in the 2nd and 3rd trimesters of pregnancy and the risk of obesity/overweight in children born to normotensive mothers. Every 10 units of increase in systolic or diastolic blood pressure were associated with a 5-8\% elevated risk of obesity. Moreover, mothers with hypertension had a $49 \%$ and $14 \%$ greater risk of overweight and obesity in their children in the 2nd and 3rd trimesters, respectively (18).

They assessed the relationship between any hypertension in the normal range and above and childhood obesity. However, according to national guidelines, hypertension can be investigated in a situation as blood pressure $\geq 140 / 90$, systolic pressure increase $>30 \mathrm{~mm} \mathrm{Hg}$, and diastolic pressure increase $>15 \mathrm{~mm} \mathrm{Hg}$ in comparison with the blood pressure taken at the first care provider visit. Therefore, the cases of preeclampsia were included according to national guidelines which resulted in the inclusion of a limited number of mothers compared to the above-mentioned study. However, similar results were obtained in the present study. Additionally, the odds ratio of obesity was higher in the current study, which can be attributed to the cumulative effects of hypertension and the lack of separation based on $10 \mathrm{~mL} / \mathrm{HG}$.

Likewise, the obtained results are consistent with the findings of Skrypnik et al indicating that an increase in pregnancy-induced hypertension leads to a $50 \%$ increase in the risk of childhood obesity (24). In their systematic review study (18 studies on 45249 cases), Davis et al also concluded that BMI increased by $0.62 \mathrm{~kg} / \mathrm{m}^{2}$ in young children born to preeclamptic mothers, regardless of gender, gestational age, and birth body weight (19), which is in line with the results of the present study. Conversely, the obtained results are not compatible with those of Kvehaugen et al (25). In the mentioned study, 23 children born to preeclamptic mothers were followed up 5-8 years after birth and then compared with the control group. The results indicated that the obesity rate was greater in the case group although this difference was not significant. This discrepancy can be ascribed to the small sample size in their study compared to the present study.

Table 3. The OR of Different Variables in the Development of Obesity in Overweight/Obese Children Aged 2-7 Years Old

\begin{tabular}{|c|c|c|c|}
\hline Variable & OR & $95 \% \mathrm{Cl}$ & $P$ value \\
\hline Gestational hypertension & 1.88 & $1.46-2.68$ & 0.014 \\
\hline History of obesity in other children & 20.58 & 4.87-86.89 & $<0.0001$ \\
\hline History of the disease in the child & 3.37 & $1.54-7.36$ & 0.002 \\
\hline History of medication use in the child & 2.99 & $1.06-8.46$ & 0.039 \\
\hline Initiation of solid food feeding before the age of 6 months & 2.39 & $1.14-5.01$ & 0.021 \\
\hline Preterm or post-term delivery & 3.06 & 1.18-7.92 & 0.021 \\
\hline Cesarean delivery & 1.46 & $0.93-2.32$ & 0.104 \\
\hline
\end{tabular}

Note. OR: Odds ratio; Cl: Confidence interval. 
Moreover, the results of this study are not in agreement with those of a prospective cohort study performed by Geelhoed et al (26). They compared mothers without hypertension, gestational hypertension, and preeclamptic, and then compared anthropometric indices at birth and found that $3.1 \%$ and $17.3 \%$ of mothers had preeclampsia and gestational hypertension, respectively. They also concluded that the odds of obesity were lower in children of preeclamptic mothers compared to those born to mothers afflicted with gestational hypertension and mothers without hypertension. In addition, the researchers controlled the positive association between the pre-pregnancy BMI and preeclampsia and child adiposity while not including high maternal BMI and high childbirth weight in the exclusion criteria. However, the two groups were homogenized in terms of confounding variables. Further, they found that the systolic and diastolic blood pressure were higher in children born to hypertensive or preeclamptic mothers in comparison to children born to mothers without gestational hypertension. Nonetheless, in the present study, the blood pressure of overweight/ obese children was within the normal range, and systolic and diastolic hypertension could not be investigated given the difference in the design of the two studies.

In the same vein, Golab et al conducted a meta-analysis of 34 prospective cohort studies and investigated 160757 mother-child couples (27). They indicated that gestational hypertension was associated with a greater chance of obesity, especially in late childhood. On the other hand, they linked preeclampsia with low BMI, especially in early childhood, which is inconsistent with the results of the present study. These researchers also controlled maternal pre-pregnancy BMI during the study. However, as previously mentioned, the effect of this factor was minimized when homogenizing case and control groups.

On the other hand, the results of the study carried out by Ehrenthal et al (28) did not confirm the results of our study. In a cohort study conducted within 20042007, they examined pre-pregnancy and prenatal factors (i.e., maternal weight gain during pregnancy, gestational diabetes, gestational hypertension, or preeclampsia) and children's BMI at the age of 4 . They did not find an association between hypertension and preeclampsia during pregnancy and increased BMI in early childhood. Moreover, they indicated that pre-pregnancy factors (i.e., maternal weight gain, gestational diabetes, and smoking) were more effective than prenatal factors. In the present study, we had no access to maternal pre-pregnancy information due to the differences in the design of the two studies. Further, the discrepancy of the results can be attributed to the ethnic diversity of the subjects and their age range (4-year-old children).

In a prospective study over a twenty-year cohort of births in a specialized gynecological hospital with a tertiary level of health care in Australia, Davis et al (29) studied 2668 young adult children whose pregnant mothers participated in Western Australian Pregnancy Cohort Study within 1991-1999. The results of their study revealed that gestational hypertension was related to a two-fold risk of obesity or overweight $(\mathrm{OR}=2,95 \%$ $\mathrm{CI}=1.5-2.8, P=0.001)$. Furthermore, children born to mothers with gestational hypertension had higher BMI regardless of their gender, gestational age, and birth body weight. The obtained odds ratio in the mentioned study agrees with the results of the present study.

In this study, $125(61 \%)$ and 80 (39\%) cases were females and males among the children in the overweight group, respectively, representing a statistically significant difference from the other group including 101 (48.3\%) and $108(51.7 \%)$ cases. This is in accordance with the results of Byberg (8) that revealed a positive correlation between exposure to preeclampsia in girls with weight and BMI. On the other hand, exposure to preeclampsia was negatively correlated with weight and BMI in infancy. However, this relationship was positive after infancy except for boys with severe preeclampsia who had lower body weights and BMI. In other words, the mentioned study investigated the differences between the two genders regarding weight and BMI in terms of severity of exposure to preeclampsia. This was one of the limitations of the present study in which the severity of preeclampsia was not achievable.

In the study conducted by Zheng et al, children were within the age range of 4-7 years with a mean and standard deviation (SD) of $5.9 \pm 0.7$ years, and the mean age of mothers at delivery was reported as $25 \pm 3.7$. In addition, the mean and SD of MBI was $20.5 \pm 2.6 \mathrm{~kg} / \mathrm{m}^{2}(18)$. The demographic characteristics of the mentioned study were comparable to those reported in the present study. Finally, Ehrenthal et al reported that the mean and SD of maternal age at delivery was $26.7 \pm 27$ (28).

\section{Conclusions}

In general, the findings of this study showed that gestational hypertension is associated with childhood obesity in the future. In this regard, prevention and control of mothers' blood pressure can be effective in preventing increased BMI in children and its consequent complications. Furthermore, some other contributing factors were identified, including the initiation of complementary food before 6 months of age and the use of gadgets such as televisions and computers for than 2 hours on a daily basis. Accordingly, the identification of the influential factors affecting childhood obesity and the necessity of preventive measures at primary and secondary levels are extremely important and among health priorities even before birth.

\section{Limitations of the Study}

The present study had some limitations. One of the limitations of this study was the single measurement of pregnant women's blood pressure on care provider visits and its retrospective nature which can be the source of 
error in data collection. Eventually, demographic variables could have affected the results since the current research was conducted in a specific geographical area.

\section{Suggestions for Further Research}

It is recommended to conduct a prospective multicenter study with a larger sample size to determine the relationship more accurately.

\section{Conflict of Interests}

Authors declare that they have no conflict of interests.

\section{Ethical Issues}

The proposal of the present study was approved by the Ethics Committee of Mazandaran University of Medical Sciences under the code of IR. MAZUMS.REC.1398.449.

\section{Financial Support}

This research was funded by the Vice Chancellor of the Mazandaran University of Medical Sciences, Sari, Iran.

\section{References}

1. World Health Organization (WHO). Taking Action on Childhood Obesity. WHO; 2018. https://apps.who.int/ iris/bitstream/handle/10665/274792/WHO-NMH-PNDECHO-18.1-eng.pdf?ua=1.

2. World Health Organization (WHO). New Global Estimates of Child and Adolescent Obesity Released on World Obesity Day. http://www.who.int/end-childhood-obesity/ en. Accessed Jan 20, 2018.

3. Kelishadi R, Haghdoost AA, Sadeghirad B, Khajehkazemi R. Trend in the prevalence of obesity and overweight among Iranian children and adolescents: a systematic review and meta-analysis. Nutrition. 2014;30(4):393-400. doi:10.1016/j.nut.2013.08.011

4. World Health Organization (WHO). Report of the Commission on Ending Childhood Obesity: Implementation Plan: Executive Summary. WHO; 2017. http://www.who.int/end-childhood-obesity/publications/ echo-plan-executive-summary/en. Accessed Jan 20, 2018.

5. Hakim J, Senterman MK, Hakim AM. Preeclampsia is a biomarker for vascular disease in both mother and child: the need for a medical alert system. Int J Pediatr. 2013;2013:953150. doi:10.1155/2013/953150

6. Myatt L, Redman CW, Staff AC, et al. Strategy for standardization of preeclampsia research study design. Hypertension. 2014;63(6):1293-1301. doi:10.1161/ hypertensionaha.113.02664

7. Turner JA. Diagnosis and management of pre-eclampsia: an update. Int J Womens Health. 2010;2:327-337. doi:10.2147/ ijwh.s8550

8. Byberg KK, Øymar K, Eide GE, Forman MR, Júlíusson $\mathrm{PB}$. Exposure to preeclampsia in utero affects growth from birth to late childhood dependent on child's sex and severity of exposure: follow-up of a nested case-control study. PLoS One. 2017;12(5):e0176627. doi:10.1371/ journal.pone.0176627

9. Druet C, Ong KK. Early childhood predictors of adult body composition. Best Pract Res Clin Endocrinol Metab. 2008;22(3):489-502. doi:10.1016/j.beem.2008.02.002

10. Ogland B, Vatten LJ, Romundstad PR, Nilsen ST, Forman MR. Pubertal anthropometry in sons and daughters of women with preeclamptic or normotensive pregnancies. Arch Dis Child. 2009;94(11):855-859. doi:10.1136/ adc. 2008.150870

11. Ong KK, Kennedy K, Castañeda-Gutiérrez E, et al. Postnatal growth in preterm infants and later health outcomes: a systematic review. Acta Paediatr. 2015;104(10):974-986. doi:10.1111/apa.13128

12. Yadav S, Rustogi D. Small for gestational age: growth and puberty issues. Indian Pediatr. 2015;52(2):135-140. doi:10.1007/s13312-015-0588-Z

13. de Boo HA, Harding JE. The developmental origins of adult disease (Barker) hypothesis. Aust N Z J Obstet Gynaecol. 2006;46(1):4-14. doi:10.1111/j.1479-828X.2006.00506.x

14. Gu Y, Lu J, Li W, et al. Joint associations of maternal gestational diabetes and hypertensive disorders of pregnancy with overweight in offspring. Front Endocrinol (Lausanne). 2019;10:645. doi:10.3389/fendo.2019.00645

15. Huang Y, Zhang W, Go K, et al. Altered growth trajectory in children born to mothers with gestational diabetes mellitus and preeclampsia. Arch Gynecol Obstet. 2020;301(1):151159. doi:10.1007/s00404-020-05436-2

16. Ryckman KK, Borowski KS, Parikh NI, Saftlas AF. Pregnancy complications and the risk of metabolic syndrome for the offspring. Curr Cardiovasc Risk Rep. 2013;7(3):217-223. doi:10.1007/s12170-013-0308-y

17. Zhang S, Wang L, Leng J, et al. Hypertensive disorders of pregnancy in women with gestational diabetes mellitus on overweight status of their children. J Hum Hypertens. 2017;31(11):731-736. doi:10.1038/jhh.2017.17

18. Zheng JS, Liu H, Ong KK, et al. Maternal blood pressure rise during pregnancy and offspring obesity risk at 4 to 7 years old: the Jiaxing birth cohort. J Clin Endocrinol Metab. 2017;102(11):4315-4322. doi:10.1210/jc.2017-01500

19. Davis EF, Lazdam M, Lewandowski AJ, et al. Cardiovascular risk factors in children and young adults born to preeclamptic pregnancies: a systematic review. Pediatrics. 2012;129(6):e1552-1561. doi:10.1542/peds.2011-3093

20. Rosiek A, Maciejewska NF, Leksowski K, RosiekKryszewska A, Leksowski $€$. Effect of television on obesity and excess of weight and consequences of health. Int J Environ Res Public Health. 2015;12(8):9408-9426. doi:10.3390/ijerph120809408

21. Talachian E. Comprehensive Guide to Integrated Healthy Baby Care. Qom: Andisheye Mandegar; 2015:135. [Persian].

22. Third Country Reference Guide for Obstetrics and Gynecology. Mothers Health Office, Family and Population Health Office, Ministry of Health, Medical Education; 2017. [Persian].

23. Valafar S. Integrated Maternal Health Care. 1st ed. Tehran: Negah Electronic Charter; 2011:26. [Persian].

24. Skrypnik D, Bogdański P, Zawiejska A, Wender-Ożegowska E. Role of gestational weight gain, gestational diabetes, breastfeeding, and hypertension in mother-to-child obesity transmission. Pol Arch Intern Med. 2019;129(4):267-275. doi:10.20452/pamw.4426

25. Kvehaugen AS, Andersen LF, Staff AC. Anthropometry 
and cardiovascular risk factors in women and offspring after pregnancies complicated by preeclampsia or diabetes mellitus. Acta Obstet Gynecol Scand. 2010;89(11):14781485. doi: $10.3109 / 00016349.2010 .500368$

26. Geelhoed JJ, Fraser A, Tilling K, et al. Preeclampsia and gestational hypertension are associated with childhood blood pressure independently of family adiposity measures: the Avon Longitudinal Study of Parents and Children. Circulation. 2010;122(12):1192-1199. doi:10.1161/ circulationaha.110.936674

27. Patro Golab B, Santos S, Voerman E, Lawlor DA, Jaddoe VWV, Gaillard R. Influence of maternal obesity on the association between common pregnancy complications and risk of childhood obesity: an individual participant data meta-analysis. Lancet Child Adolesc Health. 2018;2(11):812-821. doi:10.1016/s2352-4642(18)30273-6

28. Ehrenthal DB, Maiden K, Rao A, et al. Independent relation of maternal prenatal factors to early childhood obesity in the offspring. Obstet Gynecol. 2013;121(1):115-121. doi:10.1097/aog.0b013e318278f56a

29. Davis EF, Lewandowski AJ, Aye C, et al. Clinical cardiovascular risk during young adulthood in offspring of hypertensive pregnancies: insights from a 20year prospective follow-up birth cohort. BMJ Open. 2015;5(6):e008136. doi:10.1136/bmjopen-2015-008136

(c) 2020 The Author(s); This is an open-access article distributed under the terms of the Creative Commons Attribution License (http:// creativecommons.org/licenses/by/4.0), which permits unrestricted use, distribution, and reproduction in any medium, provided the original work is properly cited. 